\title{
Liquid Crystal Colloids Studied by THz Time-Domain Spectroscopy
}

\author{
Masahito Oh-e ${ }^{1}$, Hiroshi Yokoyama', Mattijs Koeberg', \\ Euan Hendry², Mischa Bonn' \\ ${ }^{1}$ Liquid Crystal Nano-system Project, SORST, Japan Science \& \\ Technology Agency, Tsukuba, Ibaraki, Japan \\ ${ }^{2}$ FOM Institute for Atomic and Molecular Physics, Kruislaan, \\ Amsterdam, The Netherlands
}

THz time-domain spectroscopy is used to measure the frequency dependent $(0.2-$ $2.0 \mathrm{THz}$ ) complex refractive index of a pure liquid crystal $(L C), 4^{\prime}$-n-pentyl-4-cyanobiphenyl (5CB), and its $\mathrm{LC}$ colloids with $\mathrm{SiO}_{2}$ particles. While the refractive index of the pure $L C$ is found to vary markedly due to distinct orientated domains within the sample, the LC colloids provide us with very stable and reproducible spectra, from which we can reliably deduce the optical constants of pure 5CB using effective medium theory. While the absorption coefficient is found to be very small, the refractive index of $5 C B$ decreases considerably over our probe frequency range.

Keywords: colloid; effective medium theory; dielectric; liquid crystal; optical constant; terahertz spectroscopy

Confining and dispersing soft condensed matter such as liquid crystals (LCs) has been of significant interest for more than a decade, while looking for fundamental changes in the physical properties of the material or searching for new applications. For this, colloidal particles dispersed in an LC host have attracted much attention, and also play a crucial role in LC nano-technology [1,2]. On mixing particles with a nematic LC host, an orientational topological defect is formed at the surface of a particle if the surface anchoring is sufficiently strong [3-5]. But, in addition to the local effects, one would also expect some of the bulk properties of an LC to change, since a single particle in an

Address correspondence to Masahito Oh-e, Liquid Crystal Nano-system Project, SORST, Japan Science \& Technology Agency, 5-9-9 Tokodai, Tsukuba, Ibaraki 300-2635, Japan. E-mail: oh-e@nanolc.jst.go.jp 
LC has an ability to distort the orientation of the adjacent LC molecules over relatively long range [6]. Here, we investigate such long range changes in bulk properties of a LC colloid system by investigating the low frequency $(\mathrm{THz})$ refractive index.

$\mathrm{THz}$ time-domain spectroscopy (THz-TDS) has recently emerged as a powerful spectroscopic technique to measure optical properties of materials in the $\mathrm{THz}$ frequency range [7-13]. The optical properties of LCs have been investigated in detail covering the range from UV down to microwave frequencies. At microwave frequencies, the response is dominated by molecular reorientation. In the Debye relaxation model [14] for orientational relaxation, the refractive index decreases with increasing frequency. As the frequency moves into the $\mathrm{THz}$ region, contributions from vibrations and rotations of large segments of a molecule and collective intermolecular vibrations become more important. This allows us to interpret intermolecular interaction, and hence allowing the study of molecular assembly/ packing and motion in LC phases. The published work on refractive indices of LCs in the range of $\mathrm{THz}$ frequency is, however, limited [15-19].

We report here the first application of the technique to probe the optical constants of $5 \mathrm{CB}$ in its colloids with $\mathrm{SiO}_{2}$ particles. The LC colloids are measured in the frequency range $0.2-2.0 \mathrm{THz}$ as a function of particle volume fraction. The optical constants of $5 \mathrm{CB}$ are extracted from the data of the LC colloids using effective medium theory.

The THz-TDS experimental setup has been described in detail elsewhere $[10,20]$. In brief, the $\mathrm{THz}$ pulses are generated by optical rectification in a $\langle 110\rangle$ oriented zinc telluride (ZnTe) crystal using ultrashort $(150 \mathrm{fs}, 800 \mathrm{~nm}, 1 \mathrm{kHz}$ repetition rate) pulses from a Ti:sapphire laser amplifier. Of the $2.5 \mathrm{~W}$ output power of the amplifier, $80 \mathrm{~mW}$ is used to generate $\mathrm{THz}$ pulses. These pulses are collimated and focused using nickel coated parabolic mirrors, and sent through the sample with a beam cross section of $1 \mathrm{~mm}^{2}$. The timeresolved electric field, $E(t)$, of the transmitted pulse is then detected via electro-optical effect [21] in a second $\mathrm{ZnTe}$ detection crystal. The entire spectrometer is purged by dried nitrogen to avoid water absorption.

The nematic LC used in this study is $4^{\prime}$-n-pentyl-4-cyanobiphenyl (5CB) from Merck Japan. A 2-mm thick fused-quartz cuvette, with no substrate surface treatment, is used as a container of $5 \mathrm{CB}$ and its colloids. For LC colloids, silica $\left(\mathrm{SiO}_{2}\right)$ particles with $10 \mu \mathrm{m}$ diameter from micromod Partikeltechnologie $\mathrm{GmbH}$ are dispersed in 5CB as a function of volume fraction. To mix the particles in 5CB uniformly, the mixture is appropriately shaken and sonicated before each 
measurement and the measurement is carried out before the particles precipitate.

The $\mathrm{THz}$ pulses are essentially single cycle electromagnetic pulses with a period of $\sim 1 \mathrm{ps}$. The transmission of these pulses through the cuvette with and without a sample, $E_{\text {trans }}(t)$ and $E_{r e f}(t)$, is recorder directly in the time domain. The magnitude of the field and its time delay contain the information of the absorption and the phase difference, respectively: Fourier transforming $E(t)$ gives both the amplitude $A(f)$ and the phase $\phi(f)$ of the field component in the frequency $(f)$ domain. Through the analysis of the spectral changes of the pulse introduced by the sample, the frequency dependent response of the material can be obtained. In our experiments with a thick cuvette, we can then assume the three layer model: i.e. we can regard a LC layer of thickness $d$, referred as medium 2, as being sandwiched between two fused silica plates, referred as media 1 and 3. The $\mathrm{THz}$ electric field that transmits from the medium 1 to 3 is given by $[22,23]$

$$
E_{\text {trans }}(f)=t_{12} t_{23} p(f, d) E(f),
$$

where $E(f)$ is the incident electric field, $t_{i j}$ is the transmission coefficient from medium $i$ to $j$, and $P(f, d)$ is the propagation function in medium 2 over the thickness $d$. These are defined by

$$
\begin{gathered}
t_{i j}=\frac{2 n_{i}}{n_{i}+n_{j}}, \\
P(f, d)=\exp \left(\frac{i n_{2} f d}{c}\right),
\end{gathered}
$$

where $n_{i}$ is the refractive index in medium $i$ and $c$ is the light speed in vacuum. Here, multiple reflections can be neglected in the analysis, since $d$ is very thick in our measurements. Since the electric field of the $\mathrm{THz}$ wave through an empty cuvette is described as $E_{\text {ref }}(f)=t_{1 \text { air }} t_{\text {air } 3} P_{\text {air }}(f, d) E(f)$, the difference in the complex transmission $\Delta E$ between a sample and an empty cell, divided by $E_{r e f}$, is given by

$$
\frac{\Delta E(f)}{E_{\text {ref }}(f)}=\frac{E_{\text {trans }}(f)-E_{\text {ref }}(f)}{E_{\text {ref }}(f)}=\frac{t_{12} t_{23} \exp \left(i_{2} f d / c\right)}{t_{1 \text { air }} t_{\text {air } 3} \exp (i f d / c)}-1 .
$$

In our analysis we used $n_{1}=n_{3}=1.95$ for the refractive index of the fused-silica substrate. Numerical analysis of Eq. (4) allows us to quantitatively extract the refractive index $n(f)=\operatorname{Re}\left[n_{2}(f)\right]$ and the absorption coefficient $k(f)=\operatorname{Im}\left[n_{2}(f)\right][24,25]$. 


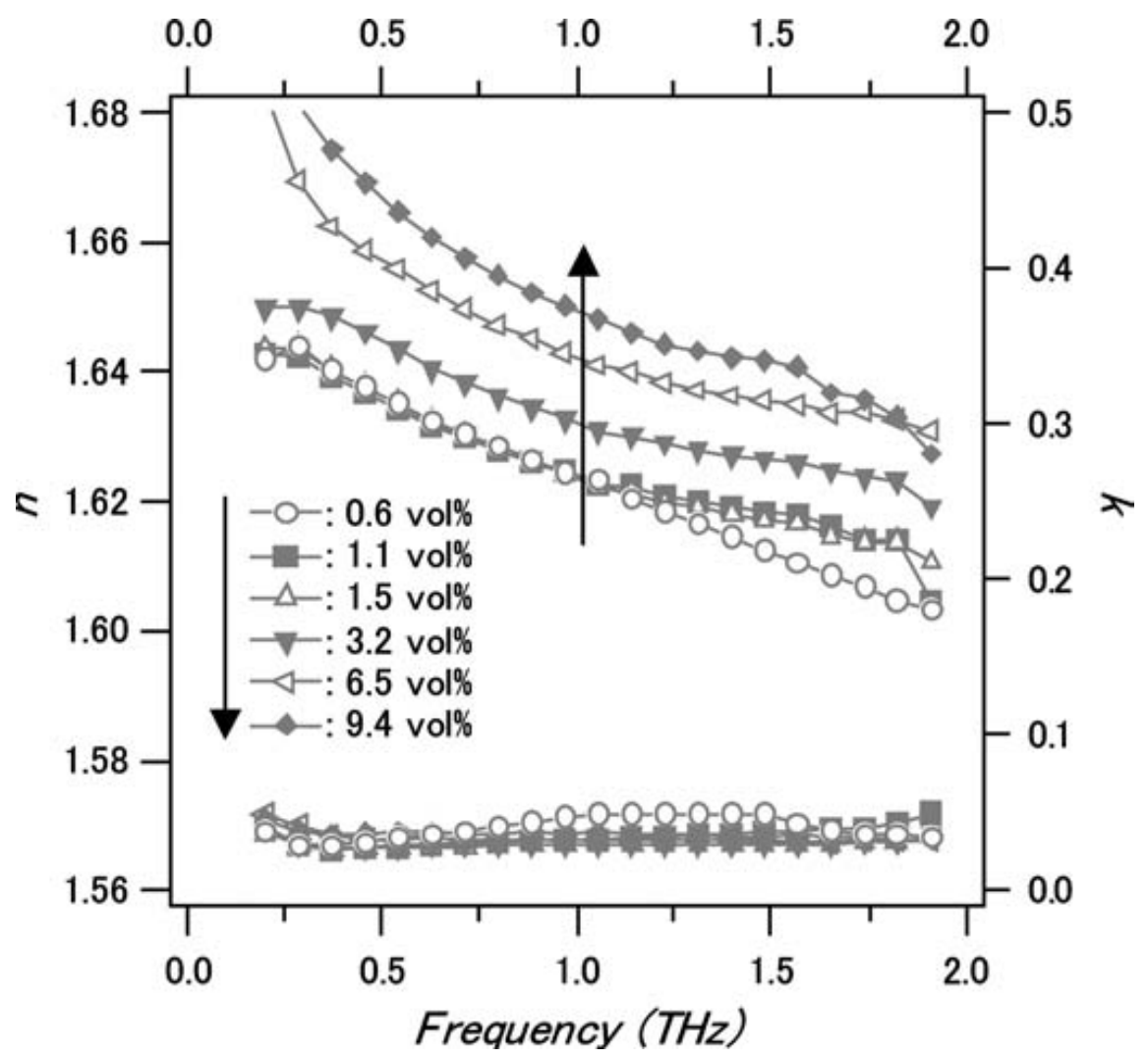

FIGURE $1 \mathrm{THz}$ frequency dependence of the refractive index $n(f)$ and the absorption coefficient $k(f)$ as a function of $\mathrm{SiO}_{2}$ particle volume fraction.

Shown in Figure 1 is the extracted refractive index $n(f)$ and absorption coefficient $k(f)$ from the measurements of LC colloids, plotted for several particle volume fractions. There is very little difference in $n(f)$ for the LC colloids of the small volume fraction of $\mathrm{SiO}_{2}$ (from $0.6 \%$ to $1.5 \%$ ), but they are within the observed reproducibility of our measurements for the pure $5 \mathrm{CB}$ without any particle due to the reason as mentioned below. For volume fractions more than $1.5 \%$, the values of $n(f)$ significantly increase. This behavior can be qualitatively explained as the adding-up effect from the higher refractive index of $\mathrm{SiO}_{2}$.

It should be pointed out that analysis of the pure $5 \mathrm{CB}$ without any particles provides us with large uncertainty, ranging from $n(f) \sim 1.60$ to $\sim 1.68$ at $1.0 \mathrm{THz}$ with a similar frequency dependence as that of the LC colloids. Looking into the reproducibility of $\mathrm{THz}$ waveforms allowed us to find an inhomogeneity in $n(f)$ for the pure 5CB. Such an inhomogeneity is shown in Figure 2, which illustrates a spread (in time) of $E_{\text {trans }}(t)$ obtained through the 5CB layer. Figure 2(b) meanwhile displays the observed fluctuation of the $\mathrm{THz}$ waves in time for different spots on the sample. While $E_{r e f}(t)$ and $E_{\text {trans }}(t)$ through the 


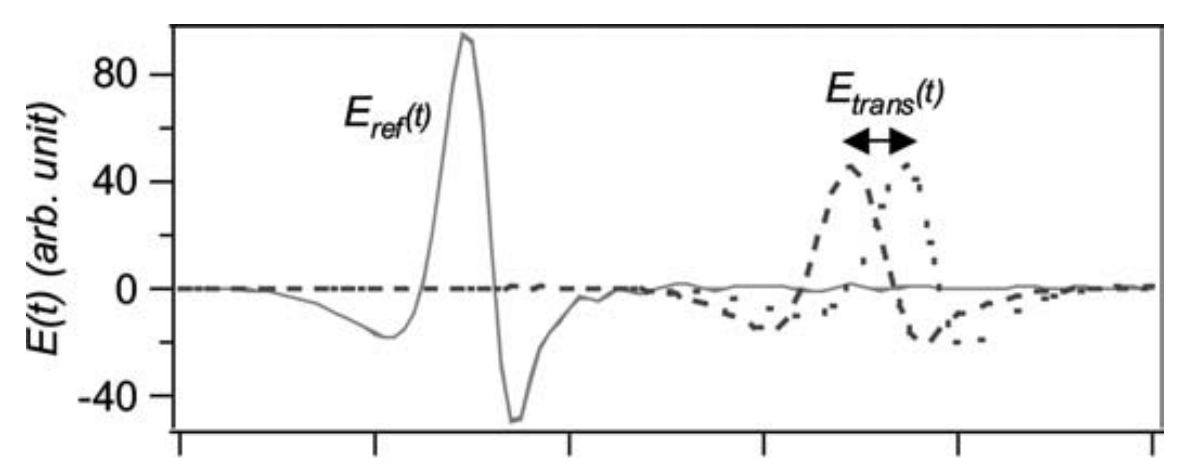

(a)

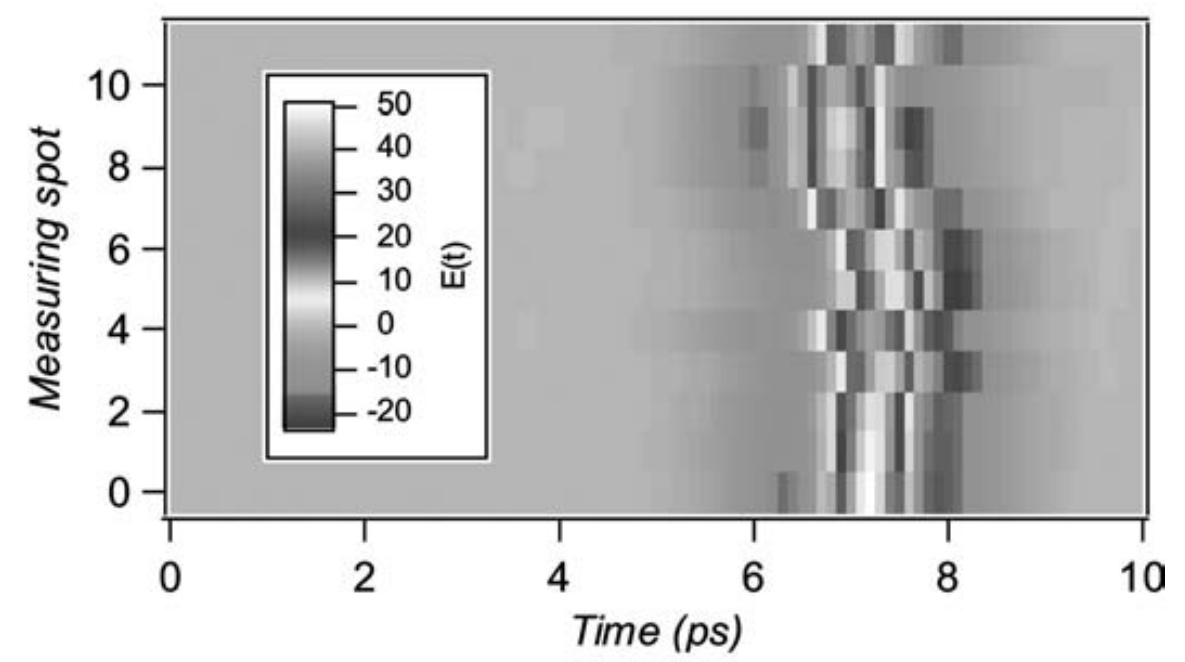

(b)

FIGURE 2 (a) THz-TDS transmitted through the cuvette with and without the 5CB layer, $E_{\text {trans }}(t)$ and $E_{r e f}(t)$. (b) Illustrated fluctuation of $E_{\text {trans }}(t)$ depending on measuring spots. The vertical axis indicates that the focusing position of the sample is changed for each one step. The brightest part corresponds to the peak of $E_{\text {trans }}(t)$.

LC colloids are very reproducible (within the $<<1 \%$ accuracy), $E_{\text {trans }}(t)$ through the pure 5CB layer can only be reproduced to within $\sim 5 \%$, determined by the repeated measurements focusing on different sampling spots. We propose that this inhomogeneity in the sample results from the birefringence of oriented LC domains formed in the LC layer, where the uniaxially oriented LC molecules at the surface induce bulk alignment by cooperatively self-organization. In our sample, the orientation of the LC molecules is clearly not homogeneous, but appears to form orientated domains. Presumably, a few LC domains of the size of tens to hundreds $\mu \mathrm{m}$ coexist within the focus area of the $\mathrm{THz}$ beam of around $\sim 1 \mathrm{~mm}$, giving rise to the observed inhomogeneity in our measurement. 
By contrast, the LC colloids gave us well reproducible $\mathrm{THz}$ wave transmission, implying that $\mathrm{SiO}_{2}$ particles introduced in the $5 \mathrm{CB}$ layer have a capability to break the LC domains and make the medium homogeneous. The particle spacing $L$ in the colloids can be estimated from the volume fraction $\alpha_{d}$ and the diameter of particle $D$ with $\alpha_{d}=\pi D^{3} / 6 L^{3}$. For the threshold particle volume observed for these effects $\left(\alpha_{d}=1.5 \sim 3.2 \%\right), L$ can be estimated to be $30 \sim 40 \mu \mathrm{m}$. This implies that the LC medium is without orientated domains as long as the distance of the particles nearby is less than $30 \sim 40 \mu \mathrm{m}$. Hence, we estimate that the domain size for the orientated segments in the pure LC is larger than $\sim 40 \mu \mathrm{m}$.

We now extract the optical constants of 5CB from those of the LC colloids shown in Figure 1. Since the sample is a mix of 5CB and $\mathrm{SiO}_{2}$ particle regions, effective medium theory can be used to extract the optical constants of 5CB. Here we use a model that assumes the particles $n_{p}$ are dispersed in a continuous host of material $n_{L C}$. For the effective refractive index $n$ of non-interacting spherical particles with index $n_{p}$ embedded within a medium $n_{L C}$, Maxwell-Garnett theory takes the form [26]

$$
\frac{n^{2}-n_{L C}^{2}}{n^{2}+2 n_{L C}^{2}}=s \frac{n_{p}{ }^{2}-n_{L C}{ }^{2}}{n_{p}^{2}+2 n_{L C}{ }^{2}},
$$

where $s$ is the space filling factor. As it neglects polarization coupling between particles Maxwell-Garnett theory is valid only for low $\mathrm{s}$ $(\mathrm{s}<0.15)[27]$ - our values of $\mathrm{s}$ are significantly lower than this limit. From Eq. (5), with $n_{p}=1.95, n$ obtained in Figure 1 and known values of $s, n_{L C}$ can be extracted. Figure 3 shows the optical constants of $5 \mathrm{CB}$ extracted from each LC colloid. The most striking feature is that $n(f)$ and $k(f)$ extracted from each LC colloid are very similar, and are in good agreement with those obtained by the average of measurements on the pure $5 \mathrm{CB}$. This suggests that any change in the $5 \mathrm{CB}$ bulk properties by foreign particles is not pronounced in the $\mathrm{THz}$ frequency range, and that the changes induced by these particles are merely orientational. Hence using LC colloids allows us to deduce the optical properties of the pure LC in a more reproducible manner by increasing the homogeneity of the sample.

The value of $n(f)$ of $5 \mathrm{CB}$ is found to decrease as a function of increasing frequency from $0.2-2 \mathrm{THz}$, while there exhibits only small absorption in this range. This is, however, in stark contrast to ref. [14], which reports that the refractive index of $5 \mathrm{CB}$ increases with increasing frequency in the range $0.3-1.4 \mathrm{THz}$. This discrepancy in the dispersion of the refractive index is probably due to complications in how the 


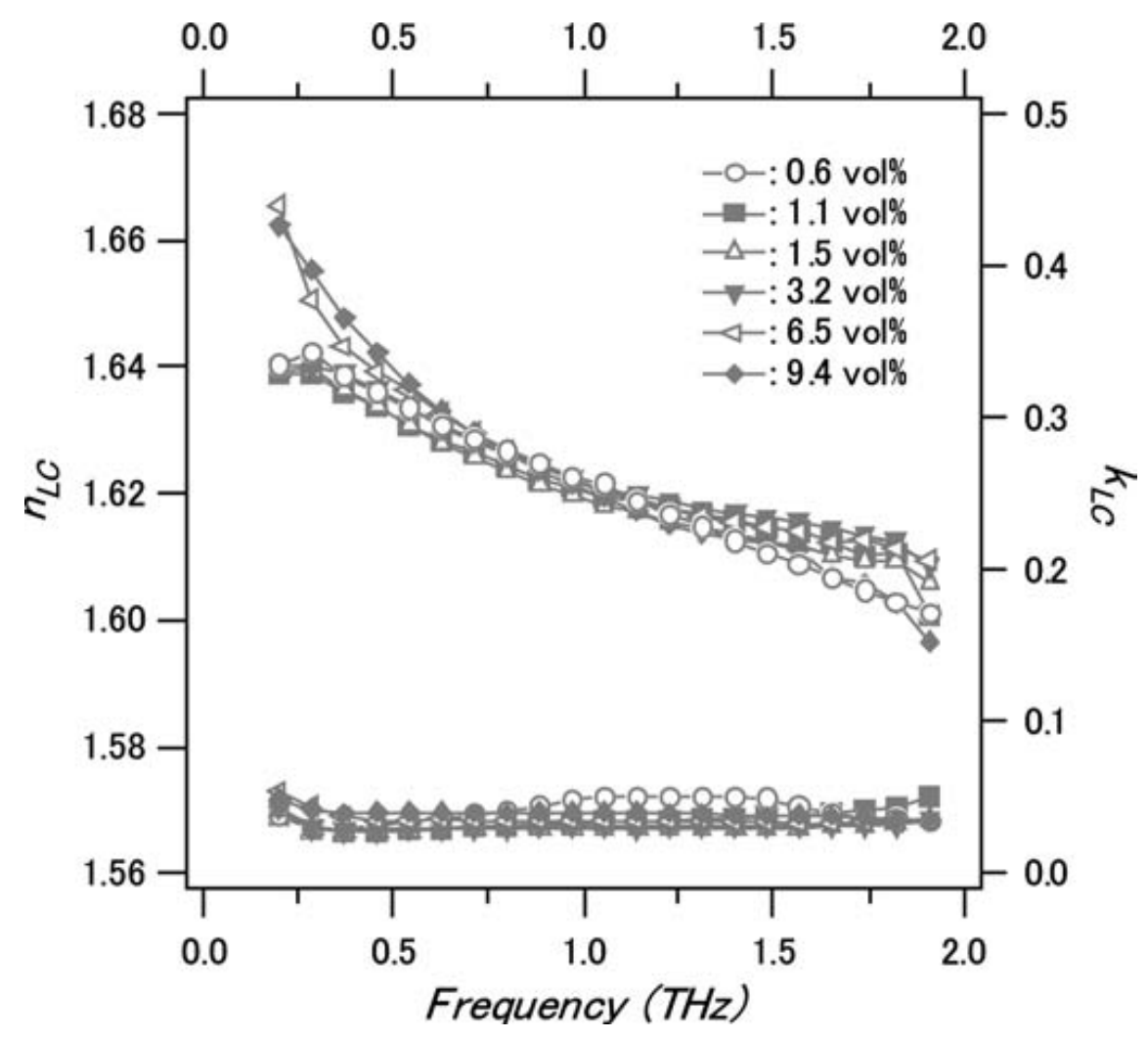

FIGURE $3 \mathrm{THz}$ frequency dependence of the refractive index $n_{L C}(f)$ and the absorption coefficient $k_{L C}(f)$ extracted using effective medium theory as a function of $\mathrm{SiO}_{2}$ particle volume fraction.

samples are prepared: in ref. [14], the very thin LC cell of $25 \mu \mathrm{m}$ used a polyimide layer to align $5 \mathrm{CB}$. Complications would presumably be caused by large interfacial effects on transmission for very thin samples, which requires precise knowledge of the interfacial dielectric properties. Other factors could be uncertainty in sample thickness and rubbing effect at the polymer surface for very thin samples. Furthermore, the dispersion that we observe is consistent with the Debye relaxation which has recently been observed in the $\mathrm{GHz}$ frequency range using the cyanobiphenyl LC by broadband dielectric spectroscopy [28]: the tail of the Debye relaxation (described by $n$ decreasing with increasing $f$ ) can reasonably be expected in the $\mathrm{THz}$ frequency range.

In conclusion, we have shown that the LC bulk optical constants can be extracted from those of the LC colloids. The extracted LC optical constants are equivalent to those of the pure LC bulk. The particles functioned as breaking LC orientation domains, giving us very stable reproducible $\mathrm{THz}$ transmission waveforms. The $\mathrm{LC}$ refractive index is found to decrease as a function of increasing frequency from 
$0.2-2 \mathrm{THz}$ with a small absorption coefficient, in line with a Debye type response of the polar material.

\section{REFERENCES}

[1] Russel, W. B., Soville, D. H., \& Schowalter, W. R. (1991). Colloidal Dispersions, Cambridge University Press: Cambridge.

[2] Drzaic, P. S. (1995). Liquid Crystal Dispersions, Series on Liquid Crystals, World Scientific: Singapore, Vol. 1.

[3] Poulin, P., Stark, H., Lubensky, T. C., \& Weitz, D. A. (1997). Science, 275, 1770.

[4] Lubensky, T. C., Pettey, D., Currier, N., \& Stark, H. (1998). Phys. Rev. E, 57, 610.

[5] Poulin, P. \& Weitz, D. A. (1998). Phys. Rev. E, 57, 626.

[6] de Gennes, P. G. \& Prost, J. (1993). The Physics of Liquid Crystals, Oxford University Press: London.

[7] Exter, M. V., Fattinger, C., \& Grischkowsky, D. (1989). Opt. Lett., 14, 1128.

[8] Grischkowsky, D., Keiding, S. R., Exter, M. V., \& Fattinger, C. (1990). J. Opt. Soc. Am. B, 7, 2006.

[9] Knoesel, E., Bonn, M., Shan, J., \& Heinz, T. F. (2001). Phys. Rev. Lett., 86, 340.

[10] Beard, M. C., Turner, G. M., \& Schmuttenmaer, C. A. (2002). J. Phys. Chem. B, 106, 7146.

[11] Shan, J., Wang, F., Knoesel, E., Bonn, M., \& Heinz, T. F. (2003). Phys. Rev. Lett., 90, 247401.

[12] Hendry, E., Schins, J. M., Candeias, L. P., et al. Phys. Rev. Lett., 92, 196601.

[13] Hendry, E., Wang, F., Shan, J., Heinz, T. F., \& Bonn, M. (2004). Phys. Rev. B 69, 081101.

[14] Gaiduk, V. I. (1999). Dielectric Relaxation and Dynamics of Polar Molecules, Series in Contemporary Chemical Physics, World Scientific: New Jersey, Vol. 8.

[15] Nose, T., Sato, S., Mizuno, K., Bae, J., \& Nozokido, T. (1997). Appl. Opt., 36, 6383.

[16] Turchinovich, D., Knobloch, P., Luessem, G., \& Koch, M. (2001). Proc. SPIE, 4463, 65.

[17] Tsai, T.-R., Chen, C.-Y., Pan, C.-L., Pan, R.-P. \& Zhang, X.-C. (2003). Appl. Opt., 42, 2372.

[18] Chen, C.-Y., Tsai, T.-R., Pan, C.-L., \& Pan, R.-P. (2003). Appl. Phys. Lett., 83, 4497.

[19] Pan, C.-L., Hsieh, C.-F., Pan, R.-P., Tanaka, M., Miyamaru, F., Tani, M., \& Hangyo, M. (2005). Opt. Express, 13, 3921.

[20] Hendry, E. (2005). PhD thesis (Leiden University, Netherlands).

[21] Planken, P. C. M., Nienhuys, H. K., Bakker, H. J., \& Wenckebach, T. (2001). J. Opt. Soc. Am. B, 18, 313.

[22] Born, M. \& Wolf, E. (1980). Principle of Optics, 6th ed. Pergamon: Oxford.

[23] Hecht, E. (2002). Optics, 4th ed. Benjamin Cummings: San Francisco.

[24] Duvillaret, L., Garet, F., \& Coutaz, J. (1996). IEEE J. Sel. Top. Quantum Electron., 2,739 .

[25] Schmuttenmaer, C. A. (2004). Chem. Rev., 104, 1759.

[26] Maxwell-Garnett, J. C. (1904). Philos. Trans. R. Soc. London, A203, 385.

[27] Niklasson, G. A., Granqvist, C. G., \& Hunderi, O. (1981). Appl. Opt., 20, 26.

[28] Leys, J., Sinha, G., Glorieux, C., \& Thoen, J. (2005). Phys. Rev. E, 71, 051709. 